\title{
ON THE EXISTENCE AND COMPACTNESS OF A TWO-DIMENSIONAL RESONANT SYSTEM OF CONSERVATION LAWS*
}

\author{
KENNETH H. KARLSEN ${ }^{\dagger}$, MICHEL RASCLE ${ }^{\ddagger}$, AND EITAN TADMOR ${ }^{\S}$
}

\begin{abstract}
We prove the existence of a weak solution to a two-dimensional resonant $3 \times 3$ system of conservation laws with $B V$ initial data. Due to possible resonance (coinciding eigenvalues), spatial $B V$ estimates are in general not available. Instead, we use an entropy dissipation bound combined with the time translation invariance property of the system to prove existence based on a two-dimensional compensated compactness argument adapted from [E. Tadmor, M. Rascle, and P. Bagnerini, J. Hyperbolic Differ. Equ., 2(3), 697-712, 2005]. Existence is proved under the assumption that the flux functions in the two directions are linearly independent.
\end{abstract}

Key words. Nonlinear conservation laws, multi-dimensional, discontinuous fluxes, entropy bounds, weak solutions, existence, compensated compactness

AMS subject classifications. 35L65, 35L 80

\section{Introduction}

This paper studies certain two-dimensional resonant $3 \times 3$ systems of conservation laws of the form

$$
\begin{aligned}
k_{t}=0, \quad l_{t} & =0, \\
u_{t}+f(k, u)_{x}+g(l, u)_{y} & =0,
\end{aligned}
$$

which are augmented with $L^{\infty} \cap B V$ initial data

$$
\left.k\right|_{t=0}=k(x, y),\left.\quad l\right|_{t=0}=l(x, y),\left.\quad u\right|_{t=0}=u_{0}(x, y) .
$$

The goal is to prove that there exists a weak solution to (1.1)-(1.2).

In recent years the one-dimensional version of the above system,

$$
\begin{aligned}
k_{t} & =0, \\
u_{t}+f(k, u)_{x} & =0,
\end{aligned}
$$

has received a considerable amount of attention. This system may be viewed as an alternative way of writing a scalar conservation law with a discontinuous flux, namely

$$
u_{t}+f(k(x), u)_{x}=0 .
$$

Equations like (1.4) occur in a variety of applications, including flow in porous media, sedimentation processes, traffic flow, radar shape-from-shading problems, blood flow, and gas flow in a variable duct.

*Received: October 2, 2006; accepted (in revised version): February 7, 2007. Communicated by Shi Jin.

${ }^{\dagger}$ Centre of Mathematics for Applications, Department of Mathematics, University of Oslo, P.O. Box 1053, Blindern, N-0316 Oslo, Norway (kennethk@math.uib.no). http://www.math.uio.no/ ${ }^{\sim}$ ken nethk/

${ }^{\ddagger}$ Laboratoire J. A. Dieudonné, UMR CNRS n. 6621, Université de Nice Sophia Antipolis, Parc Valrose, 06108 Nice Cedex 2, France (rascle@math.unice.fr). http://math1.unice.fr/ rascle/

$\S$ Department of Mathematics and Institute for Physical Science and Technology, Center of Scientific Computation And Mathematical Modeling (CSCAMM), University of Maryland, College Park, MD 20742, USA (tadmor@cscamm.umd.edu). http://www.cscamm.umd.edu/ tadmor 
If $k(x)$ is a smooth function, Kružkov's theory [22] tells us that there exists a unique entropy solution to the initial value problem for (1.4), for general flux functions $f$. The scalar Kružkov theory does not apply when $k(x)$ is discontinuous. Instead it proves useful to rewrite (1.4) as a $2 \times 2$ system of equations (1.3), which makes it possible to apply ideas from the theory of systems of conservation laws.

As a starting point, it is necessary to introduce conditions on the flux $f(k, u)$ that guarantee that solutions stay uniformly bounded. For example, one can require $f(k, a)=f(k, b)=0$ for all $k$, which in fact implies that the interval $[a, b] \subset \mathbb{R}$ becomes an invariant region. The system (1.4) has two eigenvalues, namely $\lambda_{1}=0$ and $\lambda_{2}=f_{u}(k, u)$. Consequently, if $f_{u}(k, u)$ vanishes for some value of $(k, u)$, then (1.4) is nonstrictly hyperbolic and experiences so-called nonlinear resonant behavior, which implies that wave interactions are more complicated than in strictly hyperbolic systems. As a matter of fact, one cannot expect to bound the total variation of the conserved quantities directly, but only when measured under a certain singular mapping. A singular mapping that is relevant for (1.3) is

$$
\Psi(k, u)=\int^{u}\left|f_{u}(k, \xi)\right| d \xi .
$$

If $\left\{u^{\rho}\right\}_{\rho>0}$ is a sequence of "reasonable" approximate solutions of (1.3), then one proves that the total variation of the transformed quantity $z^{\rho}:=\Psi\left(k, u^{\rho}\right)$ is bounded independently of $\rho$. Helly's theorem then gives convergence (along a subsequence) of $z^{\rho}$ as $\rho \downarrow 0$. Since the continuous mapping $u \mapsto \Psi(k, u)$ is one-to-one, $u^{\rho}$ also converges.

A singular mapping was used first by Temple [40] to establish convergence of the Glimm scheme (and thereby the existence of a weak solution) for a $2 \times 2$ resonant system of conservation laws modeling the displacement of oil in a reservoir by water and polymer, which is now known to be equivalent to a conservation law with a discontinuous coefficient (see, e.g., [21]). Since then, the singular mapping approach has been used and adapted by a great many authors to prove existence of weak solutions to resonant systems of conservation laws or scalar conservation laws with discontinuous flux functions. This was achieved by establishing convergence of various approximations schemes - Glimm and Godunov schemes, front tracking, upwind and central type schemes, vanishing viscosity/smoothing method, etc.; see (the list is far from being complete) $[1,2,3,5,6,7,9,10,11,12,13,19,20,21,23,24,30,36,41,42]$. Similar ideas have been used also in the context of degenerate parabolic equations [16]. Regarding uniqueness and entropy conditions for scalar conservation laws with discontinuous coefficients, see $[17,18]$ and the references therein.

As an alternative to the singular mapping approach, the papers $[14,15,18]$ have suggested to use the compensated compactness method and "scalar entropies" for the convergence analysis of approximate solutions. The results obtained with this approach are more general (and to some extent the proofs are easier) than those obtained with the singular mapping approach.

All the papers up to now have addressed the one-dimensional case. The aim of the present paper is to take a first look at the multi-dimensional case, which is completely unexplored. More precisely, we will prove the existence of at least one weak solution to the initial value problem for the two-dimensional system (1.1).

Our existence proof is based on studying the " $(\varepsilon, \delta) \downarrow(0,0)$ limit" of classical solutions $u^{\varepsilon, \delta}$ of the uniformly parabolic equation

$$
u_{t}^{\varepsilon, \delta}+f\left(k^{\delta}, u^{\varepsilon, \delta}\right)_{x}+g\left(l^{\delta}, u^{\varepsilon, \delta}\right)_{y}=\varepsilon\left(u_{x x}^{\varepsilon, \delta}+u_{y y}^{\varepsilon, \delta}\right), \quad \varepsilon>0, \delta>0,
$$


where $k^{\delta}, l^{\delta}$ converge to $k, l$ in $L_{\text {loc }}^{1}\left(\mathbb{R}^{2}\right)$, respectively, as $\delta \downarrow 0$.

Observe that we are essentially considering a scalar approximation scheme for (1.1); see $[6,7,16,14,15,18,41,42]$ for other scalar approximation schemes for one-dimensional discontinuous flux problems.

Although spatial $B V$ bounds are out of reach, we still have a time translation invariance property at our disposal, which, together with the assumption of $B V$ initial data, implies that $u_{t}^{\varepsilon, \delta}$ is uniformly bounded in $L^{1}$. Consider three functions $F(k, u)$, $G(l, u), H(k, l, u)$ defined by

$$
F_{u}=\left(f_{u}\right)^{2}, \quad G_{u}=\left(g_{u}\right)^{2}, \quad H_{u}=f_{u} g_{u} .
$$

We prove, at least under the assumption that $\varepsilon$ and $\delta$ are of comparable size, that the two sequences

$$
F\left(k(x, y), u^{\varepsilon, \delta}\right)_{x}+H\left(k(x, y), l(x, y), u^{\varepsilon, \delta}\right)_{y}
$$

and

$$
H\left(k(x, y), l(x, y), u^{\varepsilon, \delta}\right)_{x}+G\left(k(x, y), u^{\varepsilon, \delta}\right)_{y}
$$

are compact in $W_{\text {loc }}^{-1,2}\left(\mathbb{R}^{2}\right)$, for each fixed $t>0$.

The crux of the convergence analysis is then to prove that the above $W_{\text {loc }}^{-1,2}\left(\mathbb{R}^{2}\right)$ compactness is sufficient to establish a "two-dimensional" compensated compactness argument in the spirit of the classical Tartar-Murat results for one-dimensional conservation laws [27, 28, 29, 38, 39] (see also [4]). Here we follow the recent two-dimensional compensated compactness framework developed in Tadmor et. al. [37] for nonlinear conservation laws. We extend their results to the case involving additional discontinuous "variable coefficients". Accordingly, we make the nonlinearity assumption that for each fixed $k, l$ the functions $u \mapsto f_{u}(k, u)$ and $u \mapsto g_{u}(l, u)$ are almost everywhere linearly independent. (See (2.4) in the next section for a precise statement.) Our main existence result is based on an application of the two-dimensional compensated compactness lemma with "variable coefficients" - lemma 3.2 stated in Section 3 below. Granted the nonlinearity assumption, it then yields that (a subsequence of) $u^{\varepsilon, \delta}(\cdot, \cdot, t)$ converges in $L_{\text {loc }}^{1}\left(\mathbb{R}^{2}\right)$ to a bounded function $u(\cdot, \cdot, t)$, for a.e. $t>0$. Since $u^{\varepsilon, \delta}$ is uniformly $L^{1}$ Lipschitz continuous in time we obtain, in Section 4 below, our main Theorem 2.1, stating that $u^{\varepsilon, \delta} \rightarrow u$ in $L_{\text {loc }}^{1}\left(\mathbb{R}^{2} \times \mathbb{R}_{+}\right)$and that the limit function $u$ is a weak solution of (1.1)-(1.2).

Although we have chosen to analyze the vanishing viscosity/smoothing method, the techniques used here for that purpose can also be applied to various numerical schemes, including appropriate two-dimensional versions of the scalar finite difference schemes studied in [14, 18, 41, 42].

\section{Assumptions and statement of main results}

We start by listing the assumptions on the initial conditions $u_{0}$ and the fluxes $k, l, f, g$ that are needed for the existence result.

Regarding the initial function we assume

$$
u_{0} \in L^{\infty}\left(\mathbb{R}^{2}\right) \cap B V\left(\mathbb{R}^{2}\right), \quad a \leq u_{0} \leq b \quad \text { for a.e. in } \mathbb{R}^{2} .
$$

For the discontinuous coefficients $k, l: \mathbb{R}^{2} \rightarrow \mathbb{R}$ we assume

$$
\left\{\begin{array}{l}
k, l \in L^{\infty}\left(\mathbb{R}^{2}\right) \cap B V\left(\mathbb{R}^{2}\right), \\
\alpha \leq k, l \leq \beta \quad \text { a.e. in } \mathbb{R}^{2} .
\end{array}\right.
$$


For the flux functions $f, g:[\alpha, \beta] \times[a, b] \rightarrow \mathbb{R}$ we assume

$$
\left\{\begin{array}{l}
u \mapsto f(k, u), u \mapsto g(l, u) \in C^{2}[a, b] \text { for all } k, l \in[\alpha, \beta] ; \\
k \mapsto f(k, u), l \mapsto g(l, u) \in C^{1}[\alpha, \beta] \text { for all } u \in[a, b] .
\end{array}\right.
$$

Moreover, we make the nonlinearity assumption which excludes the possibility of $\xi_{1} f(k, u)+\xi_{2} g(l, u)$ being an affine function (in $u$ ) on any nontrivial interval for all $k, l \in[\alpha, \beta]$,

$\forall|\xi|=1$ and $k, l \in[\alpha, \beta]: \xi_{1} f(k, \cdot)+\xi_{2} g(l, \cdot) \not \equiv$ affine function on any nontrivial interval.

In its slightly stronger version, this assumption requires that $f_{u}(k, \cdot)$ and $g_{u}(l, \cdot)$ are a.e. linearly independent so that the symbol $s(\xi, k, l, u):=\xi_{1} f_{u}(k, u)+\xi_{2} g_{u}(l, u)$ satisfies

$$
\forall|\xi|=1 \text { and } k, l \in[\alpha, \beta]: \quad \operatorname{meas}\{u \mid s(\xi, k, l, u)=0\}=0 .
$$

This is a straightforward generalization of the notion of nonlinearity found in [25], in their study of kinetic formulations for nonlinear conservation laws.

Finally, we need to know that our approximate solutions stay uniformly bounded. For example, this is ensured by the assumption

$$
f(k, a), f(k, b), g(l, a), g(l, b)=0 \text { for all } k, l \in[\alpha, \beta],
$$

which implies that the interval $[a, b]$ becomes an invariant region. Of course, one can relax assumption (2.5). A sufficient condition for the invariance of the interval $[a, b]$ is that the divergence of the vector field $(x, y) \mapsto(f(k(x, y), u), g(l(x, y), u))$ is nonnegative when $u=b$ and nonpositive when $u=a$. Let us emphasize that an assumption like (2.5) is essential to our analysis; without it solutions can possess concentration effects, which is a well-known feature of, for example, linear transport equations with discontinuous coefficients.

We are now ready to state our main result.

Theorem 2.1. Suppose (2.1), (2.2), (2.3), (2.4), and (2.5) hold. Then, there exists a weak solution of the initial value problem $(1.1)-(1.2), u \in L^{\infty}\left(\mathbb{R}^{2} \times \mathbb{R}_{+}\right) \cap$ $\operatorname{Lip}\left(\mathbb{R}_{+} ; L^{1}\left(\mathbb{R}^{2}\right)\right)$, satisfying

$$
\begin{aligned}
& \int_{\mathbb{R}_{+}} \int_{\mathbb{R}}\left(u \phi_{t}+f(k(x, y), u) \phi_{x}+g(l(x, y), u) \phi_{y}\right) d x d t \\
& \quad+\int_{\mathbb{R}} u_{0}(x) \phi(x, 0) d x=0, \quad \forall \phi \in \mathcal{D}\left(\mathbb{R}^{2} \times[0, \infty)\right) .
\end{aligned}
$$

The weak solution, $u$, can be constructed as a strong $L_{\text {loc }}^{1}\left(\mathbb{R}^{2} \times \mathbb{R}_{+}\right)$-limit of classical solutions $u^{\varepsilon, \delta}$ of uniformly parabolic problems,

$$
u_{t}^{\varepsilon, \delta}+f\left(k^{\delta}, u^{\varepsilon, \delta}\right)_{x}+g\left(l^{\delta}, u^{\varepsilon, \delta}\right)_{y}=\varepsilon \Delta u^{\varepsilon, \delta},
$$

with the smoothly mollified coefficients, $k^{\delta}:=\omega_{\delta} \star k$ and $l^{\delta}:=\omega_{\delta} \star l$ (outlined in section 2.1 below).

The proof of this theorem is given in the following two sections. Note that the $B V$ assumption on the coefficients $k, l$ made in $(2.2)$ is used twice in this paper. First, it is used to prove Lipschitz regularity in time, in lemma 4.2 below; then, we use it to 
prove $W_{\text {loc }}^{-1,2}\left(\mathbb{R}^{2}\right)$ compactness of the entropy production for each fixed $t>0$ in lemma 4.4 below.

We close this section with the following summary.

REMARK 2.2. Stated differently, Theorem 2.1 shows that there exists a weak solution to the following two-dimensional scalar conservation law with discontinuous coefficients $k, l \in L^{\infty}\left(\mathbb{R}^{2}\right) \cap B V\left(\mathbb{R}^{2}\right)$ :

$$
\begin{aligned}
& u_{t}+f(k(x, y), u)_{x}+g(l(x, y), u)_{y}=0, \\
& \left.u\right|_{t=0}=u_{0} \in L^{\infty}\left(\mathbb{R}^{2}\right) \cap B V\left(\mathbb{R}^{2}\right) .
\end{aligned}
$$

We note in passing that the solution operator in this case of discontinuous "variable coefficients" is not translation invariant in space and hence the $L^{1}$-contraction property of (2.6) does not imply spatial BV compactness.

Moreover, if we let $u_{0}(\cdot) \mapsto u(t, \cdot)$ denote the mapping of $(1.1)-(1.2)$, so that $u(t, \cdot)$ is a (vanishing viscosity) weak solution constructed in theorem 2.1, then by adapting standard arguments, we can prove that the mapping is compact with respect to the $L_{\text {loc }}^{1}$ norm.

\section{A compensated compactness lemma}

In this section we prove a "two-dimensional" compensated compactness lemma. We refer to $[8,26,27,28,29,38,39]$ for background information on the compensated compactness theory. We start by recalling the celebrated div-curl lemma.

LEMma 3.1. div-curl. Let $\Omega \subset \mathbb{R}^{2}$ be an open domain and let $\rho>0$ denote a parameter taking its values in a sequence which tends to zero. Suppose $D^{\rho} \rightarrow \bar{D}, E^{\rho} \rightarrow \bar{E}$ in $\left(L^{2}(\Omega)\right)^{2}$ and $\left\{\operatorname{div} D^{\rho}\right\}_{\rho>0},\left\{\operatorname{curl} E^{\rho}\right\}_{\rho>0}$ belong to a compact subset of $W_{\mathrm{loc}}^{-1,2}(\Omega)$. Then, after extracting a subsequence if necessary, we have $D^{\rho} \cdot E^{\rho} \rightarrow \bar{D} \cdot \bar{E}$ in $\mathcal{D}^{\prime}(\Omega)$ as $\rho \downarrow 0$.

The compensated compactness lemma below is tailored for two-dimensional equations, whose spatial part involves discontinuous coefficients:

$$
f(k(x, y), v(x, y))_{x}+g(l(x, y), v(x, y))_{y} .
$$

If $g(l, u)=g(u)$ and $f(k, u)=f(u)$, then the lemma below coincides with the twodimensional result of [37, Theorem 3.1]. If we set $g=0$ then the result coincides with Tartar's compensated compactness lemma for one-dimensional scalar conservation laws with genuinely nonlinear flux $f$.

Lemma 3.2. Compensated compactness. Let $\Omega \subset \mathbb{R}^{2}$ be an open domain. Let $k, l, f, g$ be functions satisfying $(2.2),(2.3),(2.4)$, and (2.5). Suppose $\left\{v^{\rho}(x, y)\right\}_{\rho>0}$ is a sequence of measurable functions on $\Omega$ that satisfies the following two conditions:

1. There exist two finite constants $a<b$ independent of $\rho$ such that

$$
a \leq v^{\rho}(x, y) \leq b \quad \text { for a.e. }(x, y) \in \Omega .
$$

2. Let the functions $F, G, H$ be defined by

$$
F_{u}(k, u)=\left(f_{u}(k, u)\right)^{2}, \quad G_{u}(l, u)=\left(g_{u}(l, u)\right)^{2}, \quad H_{u}(k, l, u)=f_{u}(k, u) g_{u}(l, u) .
$$

We assume that the two sequences

$$
\begin{aligned}
& \left\{F\left(k(x, y), v^{\rho}\right)_{x}+H\left(k(x, y), l(x, y), v^{\rho}\right)_{y}\right\}_{\rho>0}, \\
& \left\{H\left(k(x, y), l(x, y), v^{\rho}\right)_{x}+G\left(l(x, y), v^{\rho}\right)_{y}\right\}_{\rho>0}
\end{aligned}
$$


belong to a compact subset of $W_{\mathrm{loc}}^{-1,2}\left(\mathbb{R} \times \mathbb{R}_{+}\right)$.

Then there exists a subsequence, $\left\{v^{\rho}(x, y)\right\}_{\rho>0}$, that converges a.e. to a function $v \in$ $L^{\infty}\left(\mathbb{R}^{2}\right)$, and $a \leq v(x, y) \leq b$ for a.e. $(x, y) \in \Omega$.

Proof. To simplify the notation let

$$
F^{\rho}:=F\left(k(x, y), v^{\rho}\right), \quad G^{\rho}:=G\left(l(x, y), v^{\rho}\right), \quad H^{\rho}:=H\left(k(x, y), l(x, y), v^{\rho}\right),
$$

and denote their $L^{\infty}\left(\mathbb{R} \times \mathbb{R}_{+}\right)$weak- $\star$ limits by $\bar{F}, \bar{G}, \bar{H}$, respectively. Introduce the vector fields

$$
D^{\rho}=\left(F^{\rho}, H^{\rho}\right), \quad E^{\rho}=\left(-G^{\rho}, H^{\rho}\right),
$$

and denote their respective $L^{\infty}\left(\mathbb{R} \times \mathbb{R}_{+}\right)$weak- $\star$ limits by $\bar{D}, \bar{E}$.

Thanks to (3.1), we can apply the div-curl lemma to the sequences $\left\{D^{\rho}\right\}_{\rho>0}$, $\left\{E^{\rho}\right\}_{\rho>0}$ to produce

$$
\overline{D \cdot E}=\bar{D} \cdot \bar{E} \quad \text { a.e. in } \Omega
$$

that is,

$$
\overline{H^{2}-F G}=(\bar{H})^{2}-\overline{F G},
$$

which implies

$$
\overline{(H-\bar{H})^{2}-(F-\bar{F})(G-\bar{G})}=0 .
$$

Fix $c=c(x, y) \in L^{\infty}(\Omega)$. Following [37], we now consider the function $I:[a, b] \rightarrow \mathbb{R}$ defined by

$$
\begin{aligned}
I(v)= & (H(k(x, y), l(x, y), v)-H(k(x, y), l(x, y), c))^{2} \\
& -(F(k(x, y), v)-F(k(x, y), c)) \cdot(G(l(x, y), v)-G(l(x, y), c)) .
\end{aligned}
$$

Note that

$$
\begin{aligned}
I\left(v^{\rho}\right)=( & {\left.\left[H^{\rho}-\bar{H}\right]+[\bar{H}-H(k(x, y), l(x, y), c)]\right)^{2} } \\
& -\left(\left[F^{\rho}-\bar{F}\right]+[\bar{F}-F(k(x, y), c)]\right) \cdot\left(\left[G^{\rho}-\bar{G}\right]+[\bar{G}-G(l(x, y), c)]\right) .
\end{aligned}
$$

Using this and (3.2), we compute

$$
\overline{I(v)}=(\bar{H}-H(k(x, y), l(x, y), c))^{2}-(\bar{F}-F(k(x, y), c)) \cdot(\bar{G}-G(l(x, y), c)) .
$$

By the Cauchy-Schwarz inequality we have for any $u \in[a, b]$

$$
\begin{aligned}
& (H(k(x, y), l(x, y), u)-H(k(x, y), l(x, y), c))^{2} \\
= & \left(\int_{c}^{u} f_{u}(k(x, y), \xi) g_{u}(l(x, y), \xi) d \xi\right)^{2} \\
\leq & (F(k(x, y), u)-F(k(x, y), c))(G(l(x, y), u)-G(l(x, y), c)),
\end{aligned}
$$


and hence $I(\cdot) \leq 0$ with $I(c)=0$. Thanks to (2.4), the Cauchy-Schwarz inequality in (3.4) is in fact a strict inequality. This shows that the function $I(v)$ has a strict global maximum at $v=c$.

Since $u \mapsto F(k(x, y), u)$ is strictly increasing, we can choose $c$ as

$$
c(x, y):=F^{-1}(k(x, y), \bar{F}(x, y)),
$$

so that (3.3) becomes

$$
\overline{I(v)}=(\bar{H}-H(k(x, y), l(x, y), c))^{2} .
$$

Since $I(\cdot) \leq 0$, we conclude that $\bar{H}=H(k(x, y), l(x, y), c)$, and thus $\overline{I(v)}=0$. In fact, we have $I\left(v^{\rho}\right) \rightarrow 0$ a.e. in $\Omega$.

Using the fact that $c$ is a strict maximizer of $I(v)$, we have

$$
I(v) \leq-C_{\alpha} \quad \text { whenever }|v-c|>\alpha,
$$

for some constant $C_{\alpha}>0$ that depends on $\alpha$. Consequently,

$$
\operatorname{meas}\left\{\left|v^{\rho}-\bar{u}\right|>\alpha\right\} \leq \frac{-1}{C_{\alpha}} \iint_{\Omega \cap\left\{(x, y)|| v^{\rho}(x, y)-c \mid>\alpha\right\}} I\left(v^{\rho}(x, y)\right) d x d y \rightarrow 0 \quad \text { as } \rho \downarrow 0 .
$$

Since $\alpha>0$ was arbitrary, $v^{\rho} \rightarrow c$ in measure, which in turn implies that a subsequence of $\left\{v^{\rho}\right\}_{\rho>0}$ converges to $c$ a.e. in $\Omega$.

We remark that the idea of using the Cauchy-Schwarz inequality along the lines of (3.4) for proving strong compactness can be traced back to [34, 35]

\section{Proof of Theorem 2.1}

Let $k^{\delta}, l^{\delta}, u_{0}^{\delta}$ be smooth functions converging strongly to $k, l, u_{0}$ respectively. More precisely, let $\omega_{\delta} \in C_{0}^{\infty}(\mathbb{R})$ be a nonnegative function satisfying

$$
\omega(x) \equiv 0 \quad \text { for }|x| \geq 1, \quad \iint_{\mathbb{R}^{2}} \omega(x) d x=1 .
$$

For $\delta>0$, let $\omega_{\delta}(x)=\frac{1}{\delta^{2}} \omega\left(\frac{x}{\delta}\right)$ and introduce the mollified functions

$$
k^{\delta}=\omega_{\delta} \star k, \quad l^{\delta}=\omega_{\delta} \star l .
$$

We approximate the initial data $u_{0}$ by cut-off and mollification as follows:

$$
u_{0}^{\delta}=\omega_{\delta} \star\left(u_{0} \chi_{\delta}\right),
$$

where $\chi_{\delta}(x)=1$ for $|x| \leq 1 / \delta$ and 0 otherwise. In particular, we have the estimate

$$
\left\|\left(u_{0}^{\delta}\right)_{x x}+\left(u_{0}^{\delta}\right)_{y y}\right\|_{L^{1}\left(\mathbb{R}^{2}\right)} \leq \frac{1}{\delta} \iint_{\mathbb{R}^{2}}\left(\left|\left(u_{0}^{\delta}\right)_{x}\right|+\left|\left(u_{0}^{\delta}\right)_{y}\right|\right) d x d y \leq \frac{1}{\delta}\left|u_{0}\right|_{B V\left(\mathbb{R}^{2}\right)} .
$$

Observe that for $h^{\delta}=k^{\delta}, l^{\delta}, u_{0}^{\delta}$ and $h=k, l, u_{0}$, we have $h^{\delta} \in C^{\infty}\left(\mathbb{R}^{2}\right)$ and

$$
h^{\delta} \rightarrow h \quad \text { a.e. in } \mathbb{R}^{2} \text { and in } L^{p}\left(\mathbb{R}^{2}\right) \text { for any } p \in[1, \infty) \text { as } \delta \downarrow 0 .
$$

Additionally, $u_{0}^{\delta}$ is compactly supported. 
Now let $u^{\varepsilon, \delta}$ be the unique classical solution of the uniformly parabolic equation

$$
u_{t}^{\varepsilon, \delta}+f\left(k^{\delta}, u^{\varepsilon, \delta}\right)_{x}+g\left(l^{\delta}, u^{\varepsilon, \delta}\right)_{y}=\varepsilon\left(u_{x x}^{\varepsilon, \delta}+u_{y y}^{\varepsilon, \delta}\right),
$$

with initial data $\left.u^{\varepsilon, \delta}\right|_{t=0}=u_{0}^{\delta}$. The proof proceeds through a series of lemmas, which in the end show that for each $t \in[0, T]$ a subsequence of $u^{\varepsilon, \delta}(\cdot, \cdot, t)$ converges a.e. as $\varepsilon, \delta \downarrow 0$.

Our first lemma confirms the uniform bound.

Lemma 4.1. $L^{\infty}$ bound. There exists a constant $C>0$, independent of $\varepsilon, \delta$, such that

$$
\left\|u^{\varepsilon, \delta}(\cdot, \cdot, t)\right\|_{L^{\infty}\left(\mathbb{R}^{2}\right)} \leq C, \quad \text { for all } t \in(0, T) .
$$

Proof. The proof is standard and exploits assumption (2.5) to conclude that $a \leq u^{\varepsilon, \delta}(x, y, t) \leq b$ for a.e. $(x, y, t) \in \mathbb{R}^{2} \times \mathbb{R}_{+}$.

Using that (4.2) is translation invariant in time, we can prove that $u_{t}^{\varepsilon, \delta}$ is uniformly bounded in $L^{\infty}\left(\mathbb{R}_{+} ; L^{1}\left(\mathbb{R}^{2}\right)\right)$.

Lemma 4.2. Lipschitz regularity in time. Suppose the two smoothing parameters $\varepsilon$ and $\delta$ are kept in balance in the sense that

$$
\delta=C \varepsilon, \text { for some constant } C>0 .
$$

There is a constant $C_{0}$ (which is possibly dependent on $u_{0}$ but otherwise is independent of $\varepsilon, \delta)$, such that for any $t>0$

$$
\iint_{\mathbb{R}^{2}}\left|\partial_{t} u^{\varepsilon, \delta}(\cdot, \cdot, t)\right| d x d y \leq C_{0}
$$

Proof. To prove this, set $w^{\varepsilon, \delta}=u_{t}^{\varepsilon, \delta}$. Then $w$ satisfies

$$
w_{t}^{\varepsilon, \delta}+\left(f_{u}\left(k^{\delta}, u^{\varepsilon, \delta}\right) w^{\varepsilon, \delta}\right)_{x}+\left(g_{u}\left(l^{\delta}, u^{\varepsilon, \delta}\right) w^{\varepsilon, \delta}\right)_{x}=\varepsilon\left(w_{x x}^{\varepsilon, \delta}+w_{y y}^{\varepsilon, \delta}\right) .
$$

Multiplying by $\operatorname{sign}\left(w^{\varepsilon, \delta}\right)$ gives, in the sense of distributions,

$$
\begin{aligned}
& \left|w^{\varepsilon, \delta}\right|_{t}+\left(f_{u}\left(k^{\delta}, u^{\varepsilon, \delta}\right)\left|w^{\varepsilon, \delta}\right|\right)_{x}+\left(g_{u}\left(l^{\delta}, u^{\varepsilon, \delta}\right)\left|w^{\varepsilon, \delta}\right|\right)_{y} \\
& \quad=\varepsilon\left(\left|w^{\varepsilon, \delta}\right|_{x x}+\left|w^{\varepsilon, \delta}\right|_{y y}\right)-\varepsilon \operatorname{sign}^{\prime}\left(w^{\varepsilon, \delta}\right)\left(\left(w_{x}^{\varepsilon, \delta}\right)^{2}+\left(w_{y}^{\varepsilon, \delta}\right)^{2}\right),
\end{aligned}
$$

since $f_{u}\left(k^{\delta}, u^{\varepsilon, \delta}\right) w^{\varepsilon, \delta} \operatorname{sign}^{\prime}\left(w^{\varepsilon, \delta}\right) w_{x}^{\varepsilon, \delta}=g_{u}\left(k^{\delta}, u^{\varepsilon, \delta}\right) w^{\varepsilon, \delta} \operatorname{sign}^{\prime}\left(w^{\varepsilon, \delta}\right) w_{y}^{\varepsilon, \delta}=0$. Hence

$$
\frac{d}{d t} \iint_{\mathbb{R}^{2}}\left|w^{\varepsilon, \delta}\right|(x, y, \cdot) d x d y \leq 0,
$$

which, due to (4.1) and (4.3), concludes the proof.

Thanks to the previous lemma, we also have uniform $L^{\infty}\left(\mathbb{R}_{+} ; L^{2}\left(\mathbb{R}^{2}\right)\right)$ control over $\sqrt{\varepsilon} u_{x}^{\varepsilon, \delta}$ and $\sqrt{\varepsilon} u_{y}^{\varepsilon, \delta}$.

Lemma 4.3. Entropy dissipation bound. There is a constant $C$, independent of $\varepsilon, \delta$, such that

$$
\varepsilon \iint_{\mathbb{R}^{2}}\left(\left(u_{x}^{\varepsilon, \delta}(\cdot, \cdot, t)\right)^{2}+\left(u_{y}^{\varepsilon, \delta}(\cdot, \cdot, t)\right)^{2}\right) d x d y \leq C \quad \text { for any } t>0 .
$$


Proof. Multiplying (4.2) by $u^{\varepsilon, \delta}$ and then integrating yield,

$$
\begin{aligned}
& \iint_{\mathbb{R}^{2}} \varepsilon\left(\left(u_{x}^{\varepsilon, \delta}(\cdot, \cdot, t)\right)^{2}+\left(u_{y}^{\varepsilon, \delta}(\cdot, \cdot, t)\right)^{2}\right) d x d y \\
= & -\iint_{\mathbb{R}^{2}} u u_{t}^{\varepsilon, \delta} d x d y \\
& +\iint_{\mathbb{R}^{2}}\left[\left(\int_{0}^{u^{\varepsilon, \delta}} f_{u}\left(k^{\delta}, \xi\right) d \xi\right)_{x}-\left(\int_{0}^{u^{\varepsilon, \delta}} f_{k}\left(k^{\delta}, \xi\right) d \xi\right) k_{x}^{\delta}(x, y)\right] d x d y \\
& +\iint_{\mathbb{R}^{2}}\left[\left(\int_{0}^{u^{\varepsilon, \delta}} g_{u}\left(l^{\delta}, \xi\right) d \xi\right)_{y}-\left(\int_{0}^{u^{\varepsilon, \delta}} g_{l}\left(l^{\delta}, \xi\right) d \xi\right) l_{y}^{\delta}\right] d x d y .
\end{aligned}
$$

In view of Lemmas 4.1 and 4.2 and the $B V$ regularity of the coefficients, we derive easily the uniform bound

$$
\begin{aligned}
& \varepsilon \iint_{\mathbb{R}^{2}}\left(\left(u_{x}^{\varepsilon, \delta}(\cdot, \cdot, t)\right)^{2}+\left(u_{y}^{\varepsilon, \delta}(\cdot, \cdot, t)\right)^{2}\right) d x d y \\
\leq & C\left(\left\|u_{t}^{\varepsilon, \delta}\right\|_{L^{\infty}\left(\mathbb{R}_{+} ; L^{1}\left(\mathbb{R}^{2}\right)\right)}+|k|_{B V\left(\mathbb{R}^{2}\right)}+|l|_{B V\left(\mathbb{R}^{2}\right)}\right), \quad t>0,
\end{aligned}
$$

for some constant $C$ that is dependent on $\left\|u^{\varepsilon, \delta}\right\|_{L^{\infty}\left(\mathbb{R}^{2}\right)}$ but otherwise is independent of $\varepsilon, \delta$.

Lemma 4.4. Pre-compactness at each time instant. Suppose the two parameters $\varepsilon$ and $\delta$ are kept in balance in the sense that (4.3) holds. With $F, G$, and $H$ defined in Lemma 3.2, the two sequences

$$
\begin{aligned}
& \left\{F\left(k(x, y), u^{\varepsilon, \delta}\right)_{x}+H\left(k(x, y), l(x, y), u^{\varepsilon, \delta}\right)_{y}\right\}_{\varepsilon, \delta>0} \\
& \left\{H\left(k(x, y), l(x, y), u^{\varepsilon, \delta}\right)_{x}+G\left(l(x, y), u^{\varepsilon, \delta}\right)_{y}\right\}_{\varepsilon, \delta>0}
\end{aligned}
$$

then belong to a compact subset of $W_{\mathrm{loc}}^{-1,2}\left(\mathbb{R}^{2}\right)$, for each fixed $t>0$.

Proof. Let $\phi=\phi(x, y) \in \mathcal{D}\left(\mathbb{R}^{2}\right)$, and, for each fixed $t>0$, introduce the distribution

$$
\left\langle\mathcal{L}^{\varepsilon, \delta}, \phi\right\rangle=\iint_{\mathbb{R}^{2}}\left(F\left(k(x, y), u^{\varepsilon, \delta}\right) \phi_{x}+H\left(k(x, y), l(x, y) u^{\varepsilon, \delta}\right) \phi_{y}\right) d x d y .
$$

Let us first write $\mathcal{L}^{\varepsilon, \delta}=\mathcal{L}_{1}^{\varepsilon, \delta}+\mathcal{L}_{2}^{\varepsilon, \delta}$, where

$$
\begin{aligned}
\left\langle\mathcal{L}_{1}^{\varepsilon, \delta}, \phi\right\rangle=\int & \int_{\mathbb{R}^{2}}\left(F\left(k(x, y), u^{\varepsilon, \delta}\right)-F\left(k^{\delta}(x, y), u^{\varepsilon, \delta}\right)\right) \phi_{x} d x d y \\
& \quad+\iint_{\mathbb{R}^{2}}\left(H\left(k(x, y), l(x, y), u^{\varepsilon, \delta}\right)-H\left(k^{\delta}(x, y), l^{\delta}(x, y), u^{\varepsilon, \delta}\right)\right) \phi_{y} d x d y, \\
\left\langle\mathcal{L}_{2}^{\varepsilon, \delta}, \phi\right\rangle= & \iint_{\mathbb{R}^{2}}\left(F\left(k^{\delta}(x, y), u^{\varepsilon, \delta}\right) \phi_{x}+H\left(k^{\delta}(x, y), l^{\delta}(x, y), u^{\varepsilon, \delta}\right) \phi_{y}\right) d x d y .
\end{aligned}
$$

In what follows, we let $\Omega$ denote an arbitrary but fixed bounded open subset of $\mathbb{R}^{2}$. With $\phi \in W_{0}^{1,2}(\Omega)$, we have by Hölder's inequality

$$
\left|\left\langle\mathcal{L}_{1}^{\varepsilon, \delta}, \phi\right\rangle\right| \leq C\left(\left\|k-k^{\delta}\right\|_{L^{2}(\Omega)}+\left\|k-k^{\delta}\right\|_{L^{2}(\Omega)}\right)\|\phi\|_{W_{0}^{1,2}(\Omega)} \rightarrow 0
$$


as $\delta \downarrow 0$. Thus, $\left\{\mathcal{L}_{1}^{\varepsilon, \delta}\right\}_{\varepsilon, \delta>0}$ is compact in $W^{-1,2}(\Omega)$, for each fixed $t$.

Multiplying (4.2) by $f_{u}\left(k^{\delta}(x, y), u^{\varepsilon, \delta}\right)$ yields

$$
f\left(k^{\delta}, u^{\varepsilon, \delta}\right)_{t}+F\left(k^{\delta}, u^{\varepsilon, \delta}\right)_{x}+H\left(k^{\delta}, l^{\delta}, u^{\varepsilon, \delta}\right)_{y}=I_{1}^{\varepsilon, \delta}+I_{2}^{\varepsilon, \delta}+I_{3}^{\varepsilon, \delta}+I_{4}^{\varepsilon, \delta}+I_{5}^{\varepsilon, \delta},
$$

where

$$
\begin{aligned}
& I_{1}^{\varepsilon, \delta}=\left(\varepsilon u_{x}^{\varepsilon, \delta} f_{u}\left(k^{\delta}, u^{\varepsilon, \delta}\right)\right)_{x}+\left(\varepsilon u_{y}^{\varepsilon, \delta} f_{u}\left(k^{\delta}, u^{\varepsilon, \delta}\right)\right)_{y}, \\
& I_{2}^{\varepsilon, \delta}=-\varepsilon\left(u_{x}^{\varepsilon, \delta}\right)^{2} f_{u u}\left(k^{\delta}, u^{\varepsilon, \delta}\right)-\varepsilon\left(u_{y}^{\varepsilon, \delta}\right)^{2} f_{u u}\left(k^{\delta}, u^{\varepsilon, \delta}\right), \\
& I_{3}^{\varepsilon, \delta}=-\varepsilon u_{x}^{\varepsilon, \delta} f_{u k}\left(k^{\delta}, u^{\varepsilon, \delta}\right) k_{x}^{\delta}-\varepsilon u_{y}^{\varepsilon, \delta} f_{u k}\left(k^{\delta}, u^{\varepsilon, \delta}\right) k_{y}^{\delta}, \\
& I_{4}^{\varepsilon, \delta}=F_{k}\left(k^{\delta}, u^{\varepsilon, \delta}\right) k_{x}^{\delta}+H_{k}\left(k^{\delta}, l^{\delta}, u^{\varepsilon, \delta}\right) k_{y}^{\delta}+H_{l}\left(k^{\delta}, l^{\delta}, u^{\varepsilon, \delta}\right) l_{y}^{\delta}, \\
& I_{5}^{\varepsilon, \delta}=-f_{u}\left(k^{\delta}, u^{\varepsilon, \delta}\right) f_{k}\left(k^{\delta}, u^{\varepsilon, \delta}\right) k_{x}^{\delta}-f_{u}\left(k^{\delta}, u^{\varepsilon, \delta}\right) g_{l}\left(l^{\delta}, u^{\varepsilon, \delta}\right) l_{y}^{\delta} .
\end{aligned}
$$

Hence, there is a natural decomposition of $\mathcal{L}_{2}^{\varepsilon, \delta}$ into six parts. We name the six parts $\mathcal{L}_{2,0}^{\varepsilon, \delta}, \mathcal{L}_{2,1}^{\varepsilon, \delta}, \mathcal{L}_{2,2}^{\varepsilon, \delta}, \mathcal{L}_{2,3}^{\varepsilon, \delta}, \mathcal{L}_{2,4}^{\varepsilon, \delta}$, and $\mathcal{L}_{2,5}^{\varepsilon, \delta}$.

Regarding $\mathcal{L}_{2,0}^{\varepsilon, \delta}$,

$$
\left|\left\langle\mathcal{L}_{2,0}^{\varepsilon, \delta}, \phi\right\rangle\right| \leq \tilde{C}\left\|u_{t}^{\varepsilon, \delta}\right\|_{L^{\infty}\left(\mathbb{R}_{+} ; L^{1}\left(\mathbb{R}^{2}\right)\right)}\|\phi\|_{L^{\infty}(\Omega)}, \quad \phi \in C_{0}(\Omega),
$$

which yields $\left\|\mathcal{L}_{2,0}^{\varepsilon, \delta}\right\|_{\mathcal{M}(\Omega)} \leq C$, where $\mathcal{M}(\Omega)=\left(C_{c}(\Omega)\right)^{\star}$ denotes the space of bounded measures on $\Omega$.

In view of Lemma 4.3 and the uniform boundedness of the solutions, it follows that $\left\{\mathcal{L}_{2,1}^{\varepsilon, \delta}\right\}_{\varepsilon, \delta>0}$ is compact (and in fact converges to zero) in $W^{-1,2}(\Omega)$ and $\left\|\mathcal{L}_{2,2}^{\varepsilon, \delta}\right\|_{\mathcal{M}(\Omega)} \leq$ $C$ for each fixed $t>0$.

Next, for any $\phi \in C_{c}(\Omega)$, observe that

$$
\begin{aligned}
& \left|\iint_{\mathbb{R}^{2}} I_{3}^{\varepsilon, \delta} \phi d x d y\right| \\
& \leq C\left\{\iint_{\mathbb{R}^{2}}\left|\varepsilon k_{x}^{\delta}\right|\left|k_{x}^{\delta}\right|\right\}^{\frac{1}{2}}\left\{\iint_{\mathbb{R}^{2}} \varepsilon\left(u_{x}^{\varepsilon, \delta}\right)^{2} d x d y\right\}^{\frac{1}{2}} \\
& \quad+C\left\{\iint_{\mathbb{R}^{2}}\left|\varepsilon k_{y}^{\delta}\right|\left|k_{y}^{\delta}\right|\right\}^{\frac{1}{2}}\left\{\iint_{\mathbb{R}^{2}} \varepsilon\left(u_{y}^{\varepsilon, \delta}\right)^{2} d x d y\right\}^{\frac{1}{2}} .
\end{aligned}
$$

The point here is to have $\varepsilon$ and $\delta$ in balance, so that we can ensure $\left|\varepsilon k_{x}^{\delta}\right|,\left|\varepsilon k_{y}^{\delta}\right| \leq C$. More precisely, we have $\left|\varepsilon k_{x}^{\delta}\right|,\left|\varepsilon k_{y}^{\delta}\right| \leq C \frac{\varepsilon}{\delta}$, and by choosing $\varepsilon, \delta$ according to (4.3) we achieve this balance. Consequently,

$$
\left|\iint_{\mathbb{R}^{2}} I_{3}^{\varepsilon, \delta} \phi d x d y\right| \leq C\|\phi\|_{L^{\infty}(\Omega)},
$$

and thus $\left\|\mathcal{L}_{2,3}^{\varepsilon, \delta}\right\|_{\mathcal{M}(\Omega)} \leq C$, for each fixed $t>0$.

Finally, using the $B V$ regularity of the coefficients and the boundedness of the solutions,

$$
\left|\iint_{\mathbb{R}^{2}} I_{4}^{\varepsilon, \delta} \phi d x d y\right| \leq C\|\phi\|_{L^{\infty}(\Omega)}
$$


and thus $\left\|\mathcal{L}_{2,4}^{\varepsilon, \delta}\right\|_{\mathcal{M}(\Omega)} \leq C$ for each fixed $t>0$.

$t>0$.

Similarly, $\left|\iint_{\mathbb{R}^{2}} I_{5}^{\varepsilon, \delta} \phi d x d y\right| \leq C\|\phi\|_{L^{\infty}(\Omega)}$, and thus $\left\|\mathcal{L}_{2,5}^{\varepsilon, \delta}\right\|_{\mathcal{M}(\Omega)} \leq C$ for each fixed

Summarizing, we have shown that the sequence of distributions $\left\{\mathcal{L}^{\varepsilon, \delta}\right\}_{\varepsilon, \delta>0}$ satisfies the following two properties: $\{\mathrm{i}\}$ each distribution is the sum of two terms - one is compact in $W^{-1,2}(\Omega)$ and the other one is bounded in $\mathcal{M}(\Omega)$. In addition, Lemma 4.1 implies that $\{$ ii $\}\left\{\mathcal{L}^{\varepsilon, \delta}\right\}_{\varepsilon, \delta>0}$ belongs to a bounded subset of $W^{-1, \infty}(\Omega)$. We now appeal to Murat lemma [29], which guarantees that $\left\{\mathcal{L}^{\varepsilon, \delta}\right\}_{\varepsilon, \delta>0}$ belongs to a compact subset of $W^{-1,2}(\Omega)$. This concludes the proof of the first part of the lemma, since $\Omega$ was an arbitrary bounded open subset of $\mathbb{R}^{2}$. The second part of the lemma can be proved in a similar way.

Proof. Concluding the proof of Theorem 2.1. By combining Lemmas 3.2 and 4.4, we conclude that $u^{\varepsilon, \delta}(\cdot, \cdot, t)$ is pre-compact a.e. for each $t \in[0, T]$. Together with a diagonal argument, we can prove that $u^{\varepsilon, \delta}(\cdot, \cdot, t)$ converges along a subsequence a.e. in $\mathbb{R}^{2}$ and in $L_{\text {loc }}^{1}\left(\mathbb{R}^{2}\right)$, for each fixed $t>0$. Lemma 4.2 implies that

$$
\left\|u^{\varepsilon, \delta}(\cdot, \cdot, t+\tau)-u^{\varepsilon, \delta}(\cdot, \cdot, t)\right\|_{L^{1}\left(\mathbb{R}^{2}\right)} \leq C \tau, \quad \forall \tau \in(0, T-\tau),
$$

and using this $L^{1}$ time continuity estimate together with a standard density argument show that there exists a subsequence of $\left\{u^{\varepsilon, \delta}\right\}_{\varepsilon, \delta>0}$ that converges to a limit function $u$ a.e. in $\mathbb{R}^{2} \times \mathbb{R}_{+}$and in $L_{\text {loc }}^{1}\left(\mathbb{R}^{2} \times \mathbb{R}_{+}\right)$. Moreover, the limit $u$ belongs to $L^{\infty}\left(\mathbb{R}^{2} \times\right.$ $\left.\mathbb{R}_{+}\right) \cap \operatorname{Lip}\left(\mathbb{R}_{+} ; L^{1}(\mathbb{R})\right)$.

Equipped with this strong convergence it is easy to prove that the limit $u$ is a weak solution. This concludes the proof of Theorem 2.1.

We close the paper with a couple of remarks. First, we note that the a priori bounds in Lemmas 4.1, 4.2 and 4.3, being uniform in time, enabled us to deduce precompactness at each fixed $t>0$, thus circumventing the temporal argument required in [37, Appendix A]. Second, we have herein exclusively dealt with problems that are spatially two-dimensional. A possible strategy for going beyond two dimensions is to apply the compactness framework of Panov [31, 33, 32].

Acknowledgment. The research of K. H. Karlsen was supported by an Outstanding Young Investigators Award by the Research Council of Norway. The research of E. Tadmor was supported in part by NSF grant \#DMS04-07704 and ONR Grant \#N00014-91-J-1076.

\section{REFERENCES}

[1] Adimurthi, S. Mishra and G. D. V. Gowda, Optimal entropy solutions for conservation laws with discontinuous flux-functions, J. Hyperbolic Differ. Equ., 2(4), 783-837, 2005.

[2] D. Amadori, L. Gosse and G. Guerra, Godunov-type approximation for a general resonant balance law with large data, J. Differ. Equ., 198(2), 233-274, 2004.

[3] F. Bachmann and J. Vovelle, Existence and uniqueness of entropy solution of scalar conservation laws with a flux function involving discontinuous coefficients, Comm. Partial Differ. Equ., 31(1-3), 371-395, 2006.

[4] S. Benzoni-Gavage and D. Serre, Compacité par compensation pour une classe de systèmes hyperboliques de $p \geq 3$ lois de conservation, Rev. Mat. Iberoamericana, 10(3), 557-579, 1994.

[5] S. Berres, R. Bürger and K. H. Karlsen, Central schemes and systems of conservation laws with discontinuous coefficients modeling gravity separation of polydisperse suspensions, J. Comput. and Appl. Math., 164-165, 53-80, 2004. 
[6] R. Bürger, K. H. Karlsen, C. Kligenberg and N. H. Risebro, A front tracking approach to a model of continuous sedimentation in ideal clarifier-thickener units, Nonlinear Analysis: Real World Applications, 4(3), 457-481, 2003.

[7] R. Bürger, K. H. Karlsen, N. H. Risebro and J. D. Towers, Well-posedness in BVt and convergence of a difference scheme for continuous sedimentation in ideal clarifier-thickener units, Numer. Math., 97(1), 25-65, 2004.

[8] G. Q. Chen, Compactness methods and nonlinear hyperbolic conservation laws, in Some Current Topics on Nonlinear Conservation Laws, Amer. Math. Soc., Providence, RI, 33-75, 2000.

[9] T. Gimse and N. H. Risebro, Solution of the Cauchy problem for a conservation law with a discontinuous flux function, SIAM J. Math. Anal., 23(3), 635-648, 1992.

[10] J. M. K. Hong, Part I: An extension of the Riemann problems and Glimm's method to general systems of conservation laws with source terms, Part II: A total variation bound on the conserved quantities for a generic resonant nonlinear balance laws, $\mathrm{PhD}$ thesis, University of California, Davis, 2000.

[11] E. Isaacson and B. Temple, Nonlinear resonance in inhomogeneous systems of conservation laws, in Mathematics of Nonlinear Science (Phoenix, AZ, 1989), Contemp. Math., Amer. Math. Soc., Providence, RI, 108, 63-77, 1990.

[12] E. Isaacson and B. Temple, Nonlinear resonance in systems of conservation laws, SIAM J. Appl. Math., 52(5), 1260-1278, 1992.

[13] E. Isaacson and B. Temple, Convergence of the $2 \times 2$ Godunov method for a general resonant nonlinear balance law, SIAM J. Appl. Math., 55(3), 625-640, 1995.

[14] K. H. Karlsen, C. Klingenberg and N. H. Risebro, A relaxation scheme for conservation laws with a discontinuous coefficient, Math. Comp., 73(247), 1235-1259 (electronic), 2004.

[15] K. H. Karlsen, N. H. Risebro and J. D. Towers, On a nonlinear degenerate parabolic transportdiffusion equation with a discontinuous coefficient, Electron. J. Differ. Equ., 93, 23 (electronic), 2002

[16] K. H. Karlsen, N. H. Risebro and J. D. Towers, Upwind difference approximations for degenerate parabolic convection-diffusion equations with a discontinuous coefficient, IMA J. Numer. Anal., 22(4), 623-664, 2002.

[17] K. H. Karlsen, N. H. Risebro and J. D. Towers, $L^{1}$ stability for entropy solutions of nonlinear degenerate parabolic convection-diffusion equations with discontinuous coefficients, Skr. K. Nor. Vidensk. Selsk., (3), 1-49, 2003.

[18] K. H. Karlsen and J. D. Towers, Convergence of the Lax-Friedrichs scheme and stability for conservation laws with a discontinous space-time dependent flux, Chinese Ann. Math. Ser. B, 25(3), 287-318, 2004.

[19] R. A. Klausen and N. H. Risebro, Stability of conservation laws with discontinuous coefficients, J. Differ. Equ., 157(1), 41-60, 1999.

[20] C. Klingenberg and N. H. Risebro, Convex conservation laws with discontinuous coefficients, existence, uniqueness and asymptotic behavior, Comm. Partial Differ. Equ., 20(11-12), 1959-1990, 1995.

[21] C. Klingenberg and N. H. Risebro, Stability of a resonant system of conservation laws modeling polymer flow with gravitation, J. Differ. Equ., 170(2), 344-380, 2001.

[22] S. N. Kružkov, First order quasi-linear equations in several independent variables, Math. USSR Sbornik, 10(2), 217-243, 1970.

[23] L. W. Lin, B. J. Temple and J. H. Wang, A comparison of convergence rates for Godunov's method and Glimm's method in resonant nonlinear systems of conservation laws, SIAM J. Numer. Anal., 32(3), 824-840, 1995.

[24] L. W. Lin, B. J. Temple and J. H. Wang, Suppression of oscillations in Godunov's method for a resonant non-strictly hyperbolic system, SIAM J. Numer. Anal., 32(3), 841-864, 1995.

[25] P. L. Lions, B. Perthame and E. Tadmor, A kinetic formulation of multidimensional scalar conservation laws and related equations, J. Amer. Math. Soc., 7(1), 169-191, 1994.

[26] Y. Lu, Hyperbolic conservation laws and the compensated compactness method, in Chapman \& Hall/CRC Monographs and Surveys in Pure and Appl. Math., Chapman \& Hall/CRC, Boca Raton, FL, 128, 2003.

[27] F. Murat, Compacité par compensation, Ann. Scuola Norm. Sup. Pisa Cl. Sci. (4), 5(3), 489-507, 1978.

[28] F. Murat, Compacité par compensation II, Proceedings of the International Meeting on Recent Methods in Nonlinear Analysis (Rome, 1978), Bologna, Pitagora, 245-256, 1979.

[29] F. Murat, L'injection du cône positif de $H^{-1}$ dans $W^{-1, q}$ est compacte pour tout $q<2$, J. Math. Pures Appl. (9), 60(3), 309-322, 1981.

[30] A. Noussair, Riemann problem with nonlinear resonance effects and well-balanced Godunov scheme for shallow fluid flow past an obstacle, SIAM J. Numer. Anal., 39(1), 52-72 (elec- 
tronic), 2001.

[31] E. Y. Panov, On sequences of measure-valued solutions of a first-order quasilinear equation, Mat. Sb., 185(2), 87-106, 1994.

[32] E. Y. Panov, On the strong precompactness of bounded sets of measure-valued solutions of a first-order quasilinear equation, Mat. Sb., 186(5), 103-114, 1995.

[33] E. Y. Panov, A condition for the strong precompactness of bounded sets of measure-valued solutions of a first-order quasilinear equation, Mat. Sb., 190(3), 109-128, 1999.

[34] M. Rascle, Perturbations par viscosité de certains systèmes hyperboliques non linéaires, Thèse d'Etat, Univ. Lyon 1, 1983.

[35] M. Rascle and D. Serre, Compacité par compensation et systèmes hyperboliques de lois de conservation, Applications, C. R. Acad. Sci. Paris Sér. I Math., 299(14), 673-676, 1984.

[36] N. Seguin and J. Vovelle, Analysis and approximation of a scalar conservation law with a flux function with discontinuous coefficients, Math. Models Methods Appl. Sci., 13(2), 221-257, 2003.

[37] E. Tadmor, M. Rascle and P. Bagnerini, Compensated compactness for 2D conservation laws, J. Hyperbolic Differ. Equ., 2(3), 697-712, 2005.

[38] L. Tartar, Compensated compactness and applications to partial differential equations, in Nonlinear Analysis and Mechanics: Heriot-Watt Symposium, Pitman Boston Mass, IV, 136212, 1979.

[39] L. Tartar, The compensated compactness method applied to systems of conservation laws, in Systems of Nonlinear Partial Differential Equations (Oxford, 1982), NATO Adv. Sci. Inst. Ser. C Math. Phys. Sci., Reidel, Dordrecht, 111, 263-285, 1983.

[40] B. Temple, Global solution of the Cauchy problem for a class of $2 \times 2$ nonstrictly hyperbolic conservation laws, Adv. in Appl. Math., 3(3), 335-375, 1982.

[41] J. D. Towers, Convergence of a difference scheme for conservation laws with a discontinuous flux, SIAM J. Numer. Anal., 38(2), 681-698, 2000.

[42] J. D. Towers, A difference scheme for conservation laws with a discontinuous flux-the nonconvex case, SIAM J. Numer. Anal., 39(4), 1197-1218, 2001. 